\title{
Oculofaciocardiodental syndrome
}

\author{
INSERM
}

\section{Source}

INSERM. (1999). Orphanet: an online rare disease and orphan drug data base.

Oculofaciocardiodental syndrome. ORPHA:2712

Oculo-facio-cardio-dental syndrome (OFCD) is a very rare multiple congenital anomaly syndrome characterized by dental radiculomegaly, cong enital cataract, facial dismorphism and congenital heart disease. 